\title{
Certified computations with PGD model reduction in the MsFEM framework
}

\author{
Frederic LEGOLL* and Ludovic CHAMOIN ${ }^{\dagger}$ \\ * Ecole des Ponts (Laboratoire Navier) and Inria (MATHERIALS project-team) \\ 77455 Marne la Vallée, France \\ e-mail: frederic.legoll@enpc.fr \\ $\dagger$ LMT, ENS Paris-Saclay \\ Université Paris-Saclay, France \\ e-mail: ludovic.chamoin@ens-paris-saclay.fr
}

\begin{abstract}
The Multiscale Finite Element Method (MsFEM) is a Finite Element type approach for multiscale PDEs, where the basis functions used to generate the approximation space are precomputed and are specifically adapted to the problem at hand. The computation is performed in a two-stage procedure: (i) a offline stage, in which local basis functions are computed, and (ii) a online stage, in which the global problem is solved using an inexpensive Galerkin approximation.

As for any numerical method, a crucial issue is to control the accuracy of the numerical solution provided by the MsFEM approach. In that spirit, we have recently developed an a posteriori error estimate for the global error (in energy norm) [1] as well as for quantities of interest [2], along with the associated adaptive procedure. The estimates are based on the concept of Constitutive Relation Error (CRE), which we extend to the multiscale framework.

In the present work, we investigate the additional use of model reduction inside the MsFEM strategy in order to further decrease the numerical costs. We particularly focus on the use of the Proper Generalized Decomposition (PGD) for the computation of the multiscale basis functions. PGD is a suitable tool that enables to explicitly take into account variations in geometry, material coefficients or boundary conditions. We show how to efficiently use this tool to reduce the cost for the computation of the MsFEM basis functions, and how to introduce dedicated estimates to certify the quality of the overall MsFEM solution. Numerical examples demonstrate the performance of our approach coupling PGD and MsFEM on several applications.
\end{abstract}

\section{REFERENCES}

[1] Chamoin, L. and Legoll, F. A posteriori error estimation and adaptive strategy for the control of MsFEM computations. Computer Methods in Applied Mechanics and Engineering, Vol. 336, pp. 1-38, (2018).

[2] Chamoin, L. and Legoll, F. Goal-oriented error estimation and adaptivity in MsFEM computations. arxiv preprint 1908.00367. 